\title{
Word frequencies: A comparison of Pareto type distributions
}

\author{
Martin Wiegand, Saralees Nadarajah \\ School of Mathematics, University of Manchester, Manchester M13 9PL, UK
}

July 20, 2020

\begin{abstract}
Mehri and Jamaati [Physics Letters A, 381, 2470-2477, 2017] used Zipf's law to model word frequencies in Holy Bible translations for one hundred live languages. We compare the fit of Zipf's law to a number of Pareto type distributions. A Pareto type III distribution is shown to provide the best fit, as judged by a number of comparative plots and error measures. The fit of Zipf's law appears generally poor.
\end{abstract}

Keywords: Kolmogorov-Smirnov test statistic, Squared error, Zipf's law.

\section{Introduction}

The primary means of communication among humans relies on the use of language to express ideas and emotions to one another. Depending on the language spoken, there seems to be a seemingly limitless amount of words. Strikingly certain words or word groups appear more often than others. This observation was first described by George Kingsley Zipf [23], who popularised an explanation based on the assumption that humans would use the most efficient way to describe a given concept.

Thus one would rather use specific, concise phrasing rather than a long-winded explanation with the same amount of informational value conveyed. Similar explanations had been mentioned earlier by Auerbach [4] and Jean-Baptiste Estoup as claimed by Manning and Schutze [15].

To quantify this assumption Zipf provided a power law relationship between frequency and ranked usage. This relation can be applied to a number of naturally occurring sequence frequencies,

such as medical or financial data [9], [5]. Mehri and Jamaati [18] applied Zipf's law to the word distribution of different languages based on one hundred translations of the Bible [13]. 
Zipf's Law and Pareto distributions are ubiquitous in language. They even exist when language is treated as networks: structural properties of weighted networks [17]; modeling in random texts [7]; structure-semantics interplay in complex networks [3]; statistical properties of unknown texts in the Voynich manuscript [2]; authorship recognition via fluctuation analysis of network topology [1].

We believe that the established method of a power law does not provide an appropriate fit and that the related Pareto type distributions could offer superior alternatives. After introducing the original formulation of Zipf's power law and applying it to the bi-dimensional data, we do the same for the generalized Pareto, as well as Pareto types I-III distributions. We apply the KolmogorovSmirnov (KS) test statistic along with a R squared measure and a squared error. The different fits will be visualised by a number of comparative Log-Log plots for selected languages. Additionally we ran the fitting process on all languages stated in [18], and have plotted the error measures accordingly, to visualise the effectiveness of both approaches. To verify the outcome for single author literary works we have added results on a number of different texts, as well as for random ly generated texts of different lengths (see [7]). We will conclude this note with a summary on different models and their suitability for further applications.

We would like to mention that there is a large body of work committed to understanding Zipf's law, more appropriate representations for rank frequency distributions, and why/when Zipf's law is broken. See [14], [10], [8], [12] and [21].

\section{Pareto type distributions and Bible translations}

From each translation of an identical Bible version a word frequency analysis is fashioned and words are ranked by use. Let $N_{v}$ denote vocabulary size (number of used words) and $N_{t}$ the text size (word count overall in the text). These are easily determined by tools such as [6], [22]); let $r$ and $f$ denote rank and frequency, respectively. The relative parameters are thus $r_{r}=r / N_{v}$ and $f_{r}=f / N_{t}$. At this point we like to note, that the original paper was ranking the frequencies succesively, meaning each rank was only given once and no two words could share the same value. This of course leads to a large amount of low-frequency words which occur only once or twice, covering a large range of ranks. This leads to the development of rank bands (as can be seen in plot below). We believe this to be misleading, since given a large enough data set, words with the same frequency will display a difference in ranking in the hundreds or thousands. It is easy to see, how these bands will cause the deviation error to have a certain static base error, since no distribution will capture the entire band. This obscures the results, since the differences in goodness of fit would be miniscule. We have therefore chosen to allow multiple equal rank for equal frequencies (right hand side plot). 

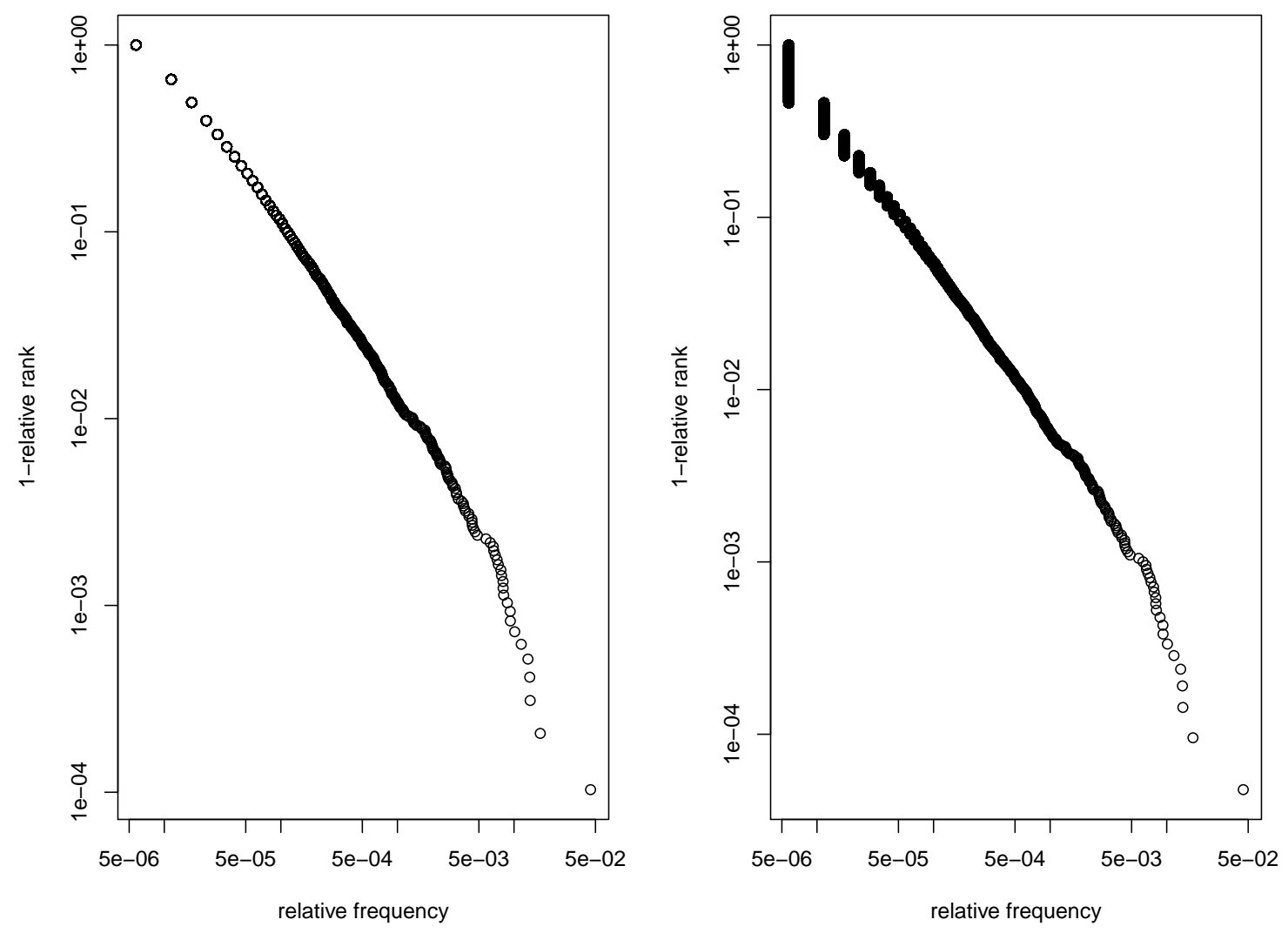

Figure 1: A comparison for the achuar language of both ranking approaches.

We seek to establish a relation similar to Zipf's relation:

$$
1-r_{r}=\exp \left[a \log \left(f_{r}\right)+b\right],
$$

where $a \in \mathbb{R}^{-}$and $b \in \mathbb{R}$. Along with this formulation we provide the performance results of the original relation provided by the Zipf law and the Zipf-Mandelbrot version given below.

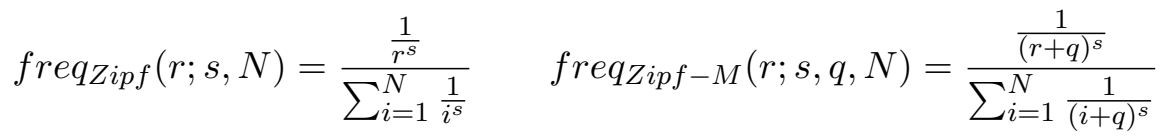

Here $r \in \mathbb{N}$ is the absolute rank and $s, q$ the respective relation parameters.

As we will see in later plots, this relationship manifests itself as a straight line in a $\log -\log$ plot. Especially in both lower and upper tails the distribution does not accurately capture the expected ranking. Down below we have listed the cumulative density functions (CDFs) of the tested Pareto-type distributions and the tested relationships between relative rank and frequency: 


$$
\begin{array}{cc}
F_{P-I}(x)=1-\left[\frac{x}{\sigma}\right]^{-\alpha}, & r_{r}=\left[\frac{f_{r}}{\sigma}\right]^{-\alpha}, \\
F_{P-I I}(x)=1-\left[1+\frac{x-\mu}{\sigma}\right]^{-\alpha}, & r_{r}=\left[1+\frac{f_{r}-\mu}{\sigma}\right]^{-\alpha}, \\
F_{P-I I I}(x)=1-\left[1+\left(\frac{x-\mu}{\sigma}\right)^{1 / \gamma}\right]^{-1}, & r_{r}=\left[1+\left(\frac{f_{r}-\mu}{\sigma}\right)^{1 / \gamma}\right]^{-1},
\end{array}
$$

and

$$
F_{P G P D}(x)=1-\left[1+\xi\left(\frac{x-\mu}{\sigma}\right)\right]^{-1 / \xi}, \quad r_{r}=\left[1+\xi\left(\frac{f_{r}-\mu}{\sigma}\right)\right]^{-1 / \xi}
$$

The parameters $\alpha>0, \gamma>0$ and $-\infty<\xi<\infty$ control the shape of these distributions. The parameter $\sigma>0$ controls the scale of these distributions. The parameter $-\infty<\mu<\infty$ controls the location of these distributions. Smaller values of $\alpha$ correspond to heavier tails of the Pareto type I-III distributions. Larger values of $\gamma$ correspond to heavier tails of the Pareto type III distribution. The generalized Pareto distribution has a finite tail if $\xi<0$. It has an infinite tail if $\xi \geq 0$. The exponential distribution is the limiting case of the generalized Pareto distribution for $\xi \rightarrow 0$. The Pareto type II distribution is the particular case of the Pareto type III distribution for $\gamma=1$. The Pareto type II distribution is a location scale variant of the Pareto type I distribution.

The four Pareto-type distributions and Zipf's law were fitted to the data by the minimization of the square deviation of the projected relative rank through a function of the relative frequency to the observed relative rank. Since the relation which was observed in the original paper was based on the CDFs of distributions, this was a more direct approach than density based approaches, say the MLE for example. We therefore provide the aggregated squared error, the KolmogorovSmirnov statistic as well as the $R^{2}$ measure on untransformed data, to diversify our measures. The squared error was minimized using the routine optim in the $\mathrm{R}$ software [19]. The routine uses a quasi Newton algorithm. The log-transformations are not considered at this point, that is during optimization. The logarithmic data is used solely to provide a better display of performances. 

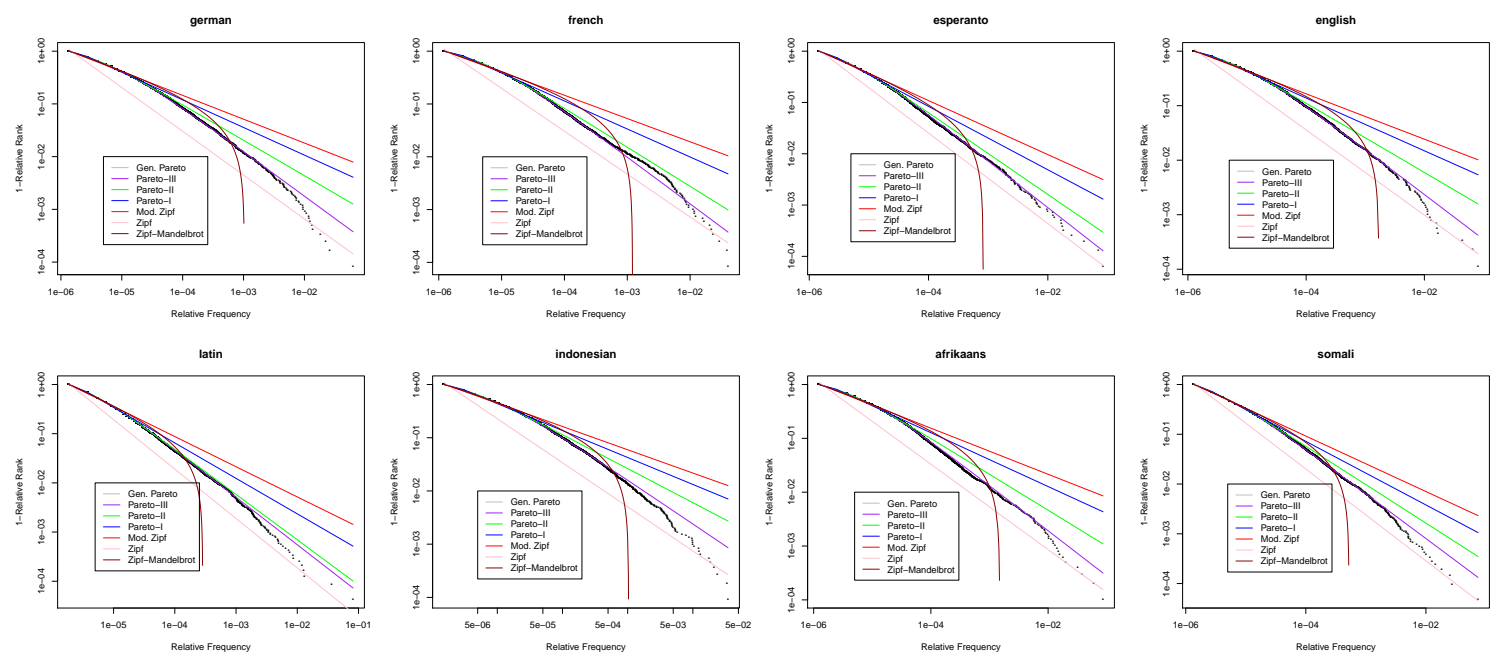

Figure 2: Log-Log inverse CDF plot of word relative frequency versus relative rank.
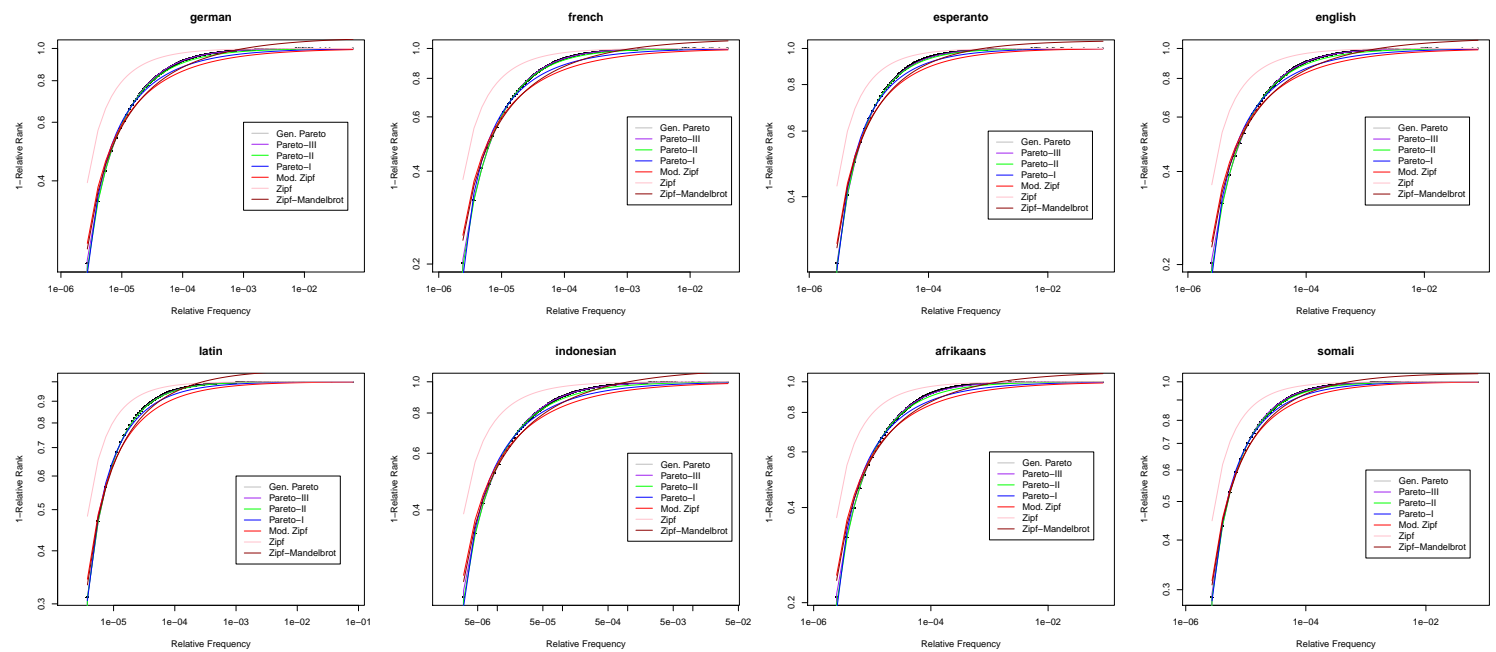

Figure 3: Log-Log CDF plot of word relative frequency versus relative rank.

The fit of the distributions for eight exemplary translations are shown in Figures 2 and 3. The picture is similar for all other languages. Zipf's method (red) does not adequately cover the curvature of the word distribution, whereas all other Pareto distributions offer a greater fit. The Pareto type III distribution repeatedly exhibits a squared error less than 0.5 thus providing the best overall performance, see Table 1, in Table 2 for further texts and in Table 3 for results by language family. Generalised and Pareto type II distributions have a slightly worse performance, yet seem to offer better results for certain languages (here Somali) and when considering the KS statistic. We have added the measure multiplied by the number of their parameters, to penalize for their complexity, yet the results remain the same, since the improvements of fit are to vast to be influenced by the different number of parameters. 


\begin{tabular}{|c|c|c|c|c|c|}
\hline & Distribution & KS statistic & Squared Error & $\mathbf{R}$ squared & Sq. Error $\mathrm{x}$ DoF \\
\hline \multirow{7}{*}{ 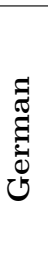 } & Gen. Pareto Dist. & 0.012941 & 0.4847 & 0.9997585 & 1.4541 \\
\hline & Pareto Dist. Type III & 0.007695 & 0.2879 & 0.9998566 & 0.8636 \\
\hline & Pareto Dist. Type II & 0.012939 & 0.4847 & 0.9997585 & 1.4541 \\
\hline & Pareto Dist. Type I & 0.036879 & 4.4943 & 0.9977609 & 8.9888 \\
\hline & Zipf-Mandelbrot Law & 0.066079 & 7.8892 & 0.9960695 & 15.7785 \\
\hline & Zipf's Power Law (mod.) & 0.062875 & 16.0795 & 0.9919891 & 32.1590 \\
\hline & Zipf's Power Law (orig.) & 0.232340 & 415.5079 & 0.7929907 & 415.5079 \\
\hline \multirow{7}{*}{ 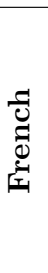 } & Gen. Pareto Dist. & 0.011481 & 0.2173 & 0.9998815 & 0.6517 \\
\hline & Pareto Dist. Type III & 0.008465 & 0.2183 & 0.9998809 & 0.6518 \\
\hline & Pareto Dist. Type II & 0.011485 & 0.2173 & 0.9998815 & 0.6517 \\
\hline & Pareto Dist. Type I & 0.042907 & 5.8038 & 0.9968332 & 11.6075 \\
\hline & Zipf-Mandelbrot Law & 0.058310 & 15.6081 & 0.9914835 & 31.21628 \\
\hline & Zipf's Power Law (mod.) & 0.076270 & 25.2547 & 0.9862199 & 50.5094 \\
\hline & Zipf's Power Law (orig.) & 0.236336 & 434.2414 & 0.7630579 & 434.2414 \\
\hline \multirow{7}{*}{ 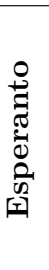 } & Gen. Pareto Dist. & 0.010794 & 0.2820 & 0.9999024 & 0.8460 \\
\hline & Pareto Dist. Type III & 0.005557 & 0.0264 & 0.9999909 & 0.0791 \\
\hline & Pareto Dist. Type II & 0.010795 & 0.2820 & 0.9999024 & 0.8460 \\
\hline & Pareto Dist. Type I & 0.032084 & 3.7774 & 0.9986926 & 7.5548 \\
\hline & Zipf-Mandelbrot Law & 0.0457038 & 11.0022 & 0.9961921 & 22.0044 \\
\hline & Zipf's Power Law (mod.) & 0.060283 & 18.0983 & 0.9937361 & 36.1966 \\
\hline & Zipf's Power Law (orig.) & 0.195324 & 415.5079 & 0.8602944 & 403.6524 \\
\hline \multirow{7}{*}{ 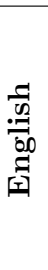 } & Gen. Pareto Dist. & 0.015998 & 0.5933 & 0.9995604 & 1.7799 \\
\hline & Pareto Dist. Type III & 0.008585 & 0.1792 & 0.9998672 & 0.5377 \\
\hline & Pareto Dist. Type II & 0.015996 & 0.5933 & 0.9995604 & 1.7799 \\
\hline & Pareto Dist. Type I & 0.043663 & 4.7441 & 0.9964850 & 9.4882 \\
\hline & Zipf-Mandelbrot Law & 0.062942 & 7.9992 & 0.9940733 & 15.9984 \\
\hline & Zipf's Power Law (mod.) & 0.070629 & 14.8486 & 0.9889985 & 29.6971 \\
\hline & Zipf's Power Law (orig.) & 0.241482 & 326.2466 & 0.7582786 & 326.2466 \\
\hline \multirow{7}{*}{ 㞼 } & Gen. Pareto Dist. & 0.063925 & 0.1266 & 0.9999746 & 0.3797 \\
\hline & Pareto Dist. Type III & 0.065341 & 0.0375 & 0.9999925 & 0.1125 \\
\hline & Pareto Dist. Type II & 0.063925 & 0.1266 & 0.9999746 & 0.3797 \\
\hline & Pareto Dist. Type I & 0.023692 & 3.1248 & 0.9993729 & 6.2496 \\
\hline & Zipf-Mandelbrot Law & 0.067431 & 9.5414 & 0.9980852 & 19.0827 \\
\hline & Zipf's Power Law (mod.) & 0.052449 & 20.6222 & 0.9958614 & 41.2445 \\
\hline & Zipf's Law (orig.) & 0.187220 & 571.0397 & 0.8853997 & 571.0397 \\
\hline \multirow{7}{*}{ 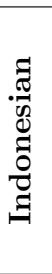 } & Gen. Pareto Dist. & 0.016838 & 0.5856 & 0.9996690 & 1.7569 \\
\hline & Pareto Dist. Type III & 0.010506 & 0.4492 & 0.9997461 & 1.3476 \\
\hline & Pareto Dist. Type II & 0.016836 & 0.5856 & 0.9996690 & 1.7569 \\
\hline & Pareto Dist. Type I & 0.039726 & 3.9837 & 0.9977487 & 7.9674 \\
\hline & Zipf-Mandelbrt Law & 0.074594 & 6.0653 & 0.9965722 & 12.1306 \\
\hline & Zipf's Power Law (mod.) & 0.065197 & 14.8299 & 0.9916190 & 29.6599 \\
\hline & Zipf's Power Law (orig.) & 0.235931 & 412.3725 & 0.7669506 & 412.3725 \\
\hline \multirow{7}{*}{ 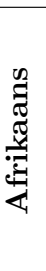 } & Gen. Pareto Dist. & 0.019155 & 0.7884 & 0.9994930 & 2.3651 \\
\hline & Pareto Dist. Type III & 0.009418 & 0.2494 & 0.9998396 & 0.7481 \\
\hline & Pareto Dist. Type II & 0.019036 & 0.7882 & 0.9994931 & 2.3651 \\
\hline & Pareto Dist. Type I & 0.047467 & 5.7356 & 0.9963114 & 11.4711 \\
\hline & Zipf-Mandelbrot Law & 0.061464 & 9.8136 & 0.9936887 & 19.6273 \\
\hline & Zipf's Power Law (mod.) & 0.074162 & 17.4008 & 0.9888094 & 34.8016 \\
\hline & Zipf's Law (orig.) & 0.238492 & 356.1892 & 0.7709310 & 356.1892 \\
\hline \multirow{7}{*}{ 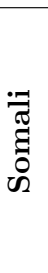 } & Gen. Pareto Dist. & 0.007878 & 0.1362 & 0.9999664 & 0.4086 \\
\hline & Pareto Dist. Type III & 0.008510 & 0.4814 & 0.9998814 & 1.9255 \\
\hline & Pareto Dist. Type II & 0.007877 & 0.1362 & 0.9999664 & 0.4086 \\
\hline & Pareto Dist. Type I & 0.022617 & 2.2622 & 0.9994425 & 4.5244 \\
\hline & Zipf-Mandelbrot Law & 0.047973 & 7.3160 & 0.9981971 & 14.6320 \\
\hline & Zipf's Power Law (mod.) & 0.045634 & 14.6678 & 0.9963854 & 29.3356 \\
\hline & Zipf's Power Law (orig.) & 0.186582 & 503.0458 & 0.8760347 & 503.0458 \\
\hline
\end{tabular}

Table 1: Kolmogorov-Smirnov statistic, squared error value and the $\mathrm{R}$ squared value for the eight selected languages. 

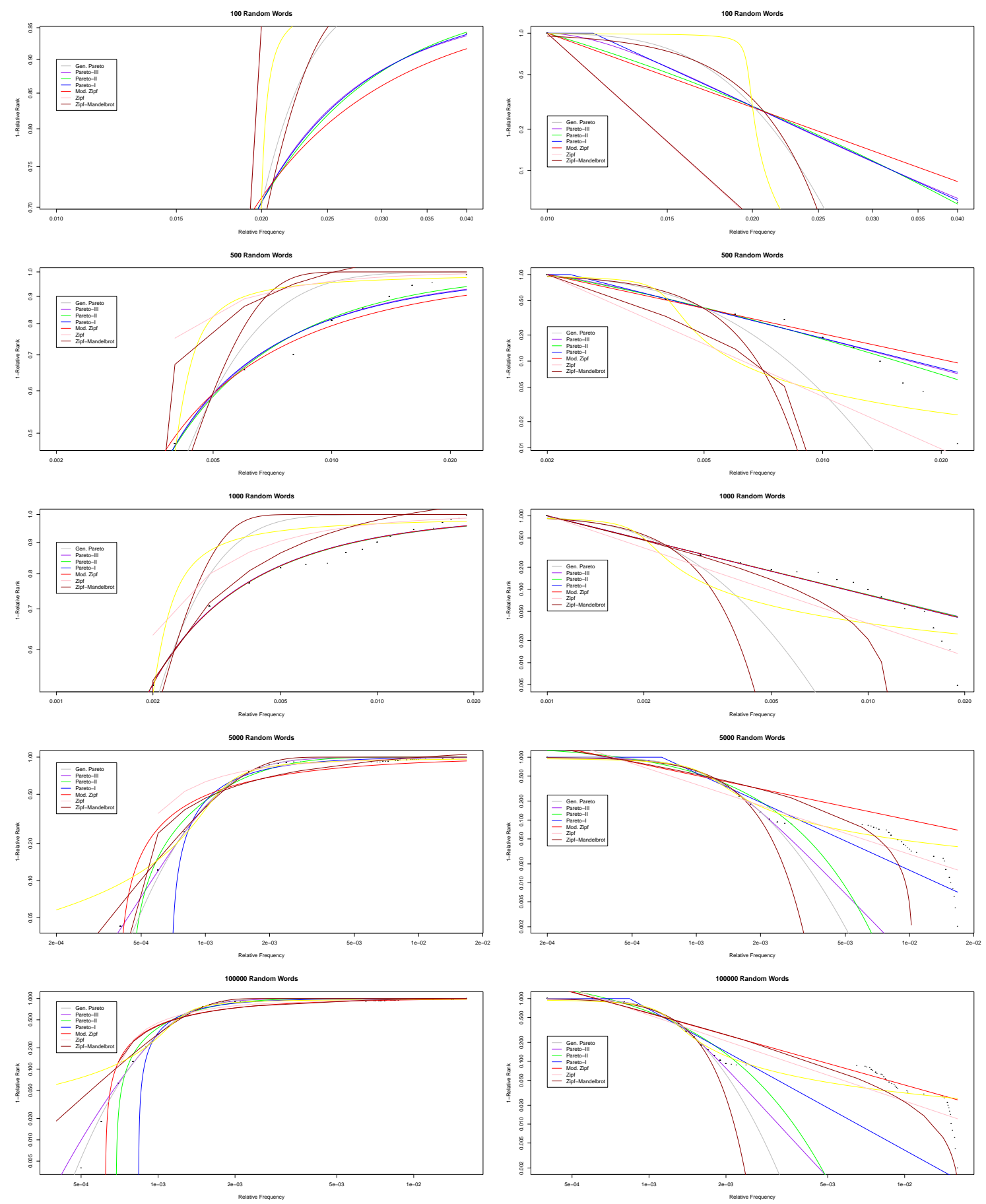

Figure 4: CDF and Inverse CDF for randomly generated texts of differents lengths.

The KS statistic, squared error value and $R^{2}$ value are shown in Table 1 for the eight selected languages. In all instances of the KS test of the Pareto type III distribution stays within the test limits. The standard Pareto distribution shows the largest deviation amongst the Pareto type distributions, yet still outperforms Zipf's law.

The plots in Figures 2 and 3 show the results for all one hundred languages, and provide further 


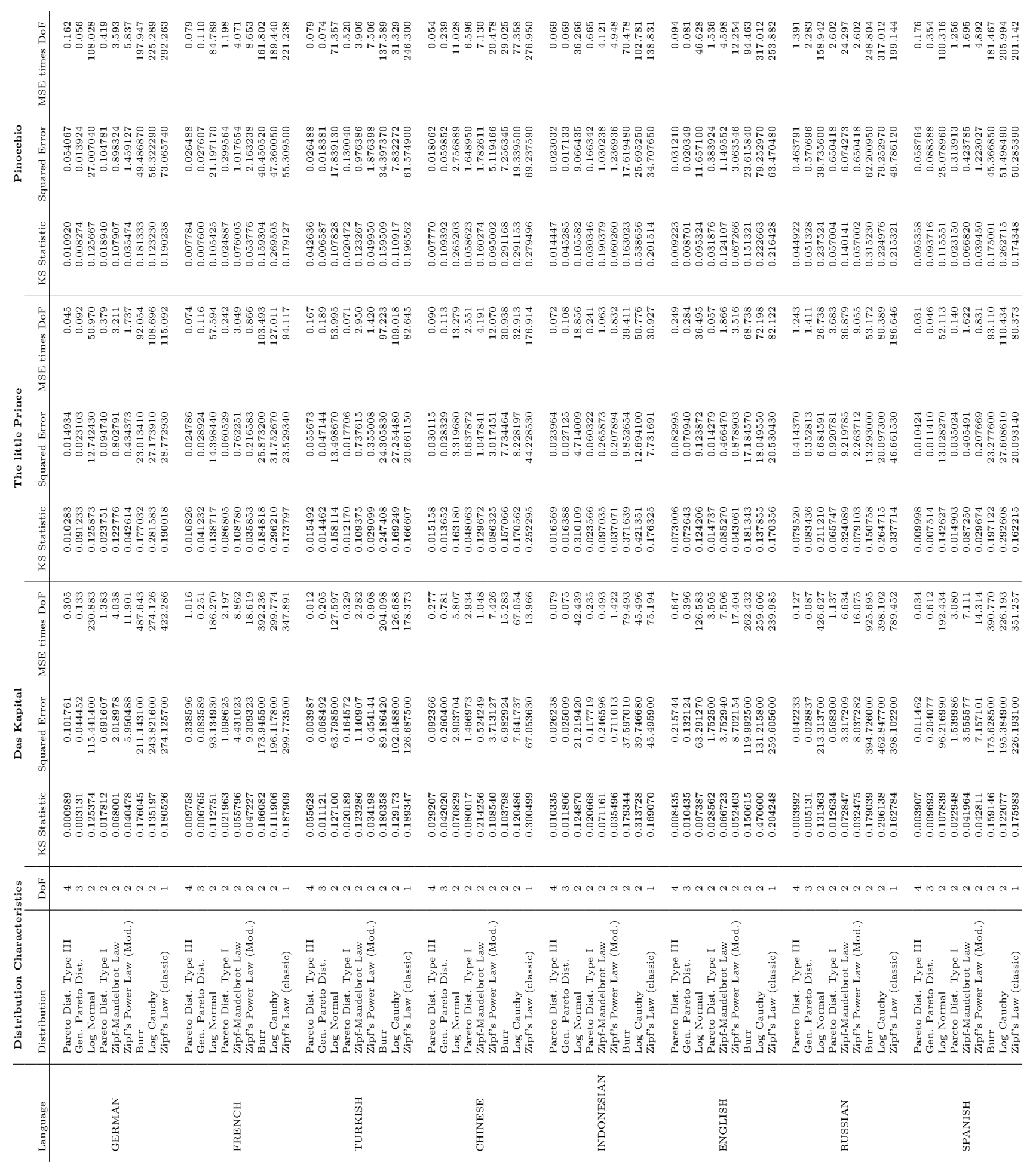

Table 2: Error measures for three single author literary works.

evidence for what we had already assumed. . Please note that in this plot the generalized Pareto II and generalized Pareto distribution overlap, and show identical results in the comparative tables. This is due to the fact that both distributions can be emulated by each other by $\alpha=1 / \xi$ and 
$\sigma=\sigma / \xi$, respectively. In Figure 5 notice that the Pareto type III distribution provides a tight grouping around 0 and 1 for the $\mathrm{KS}$ test statistic and the $\mathrm{R}$ squared measure, respectively. The Pareto type III distribution outperforms Zipf's law almost without fail.

As adressed by Ferrer-i-Cancho and Elvevå[7] we have observed the plausibility of the ability of Zipf's law to describe word frequencies. The results can be found in figure 4, as well as in a table in the Appendix. We can see an overall decline in the performance of all distributions, since the generated texts display distinct plateaus in their frequency distribution, which cannot be acurately covered by any distribution we have investigated.

However, we can confirm the findings of the referenced paper, and once again we can see similar behaviors of the distributions shaping up, as they did with the real life data. Especially the higher rates of simulation show how the Pareto-type distributions outperform the standard and modified Zipf laws.

To verify our findings, and prove the general validity of the conclusions we reach, we have repeated the procedure for a representative selection of different languages with additional single author texts, available in a number of translations (see [11],[20] and [16]).

The results are summarized in Table 2, listing the goodness of fit of every distribution investigated to each one of the literary works in question. We have tried to incorporate different subject matters and lengths of texts, to further diversify the type of text. Additionally the appendix offers similar comparative plots as for the bible translations.

For all three subsequent texts, we can see the same picture as for both the bible translations and random texts.

In Table 3 we have provided a data table of the results, grouped by language family along with a listing of the respective groupings. The overall results mirror our previous findings, but we can find aberrations for specific groups of languages. An example are the altaic languages, where the Pareto II distribution performs severly worse than the Zipf-Mandelbrot law. For for the japonic languages (japanese) and chibchuan we see a strong performance of the logarithmic distributions and the original Zipf law, which stand out distinctly from the remaining findings. These remain isolated cases though, and the Pareto III distribution specifically never completely fails to capture the behaviour of any given language translation. 


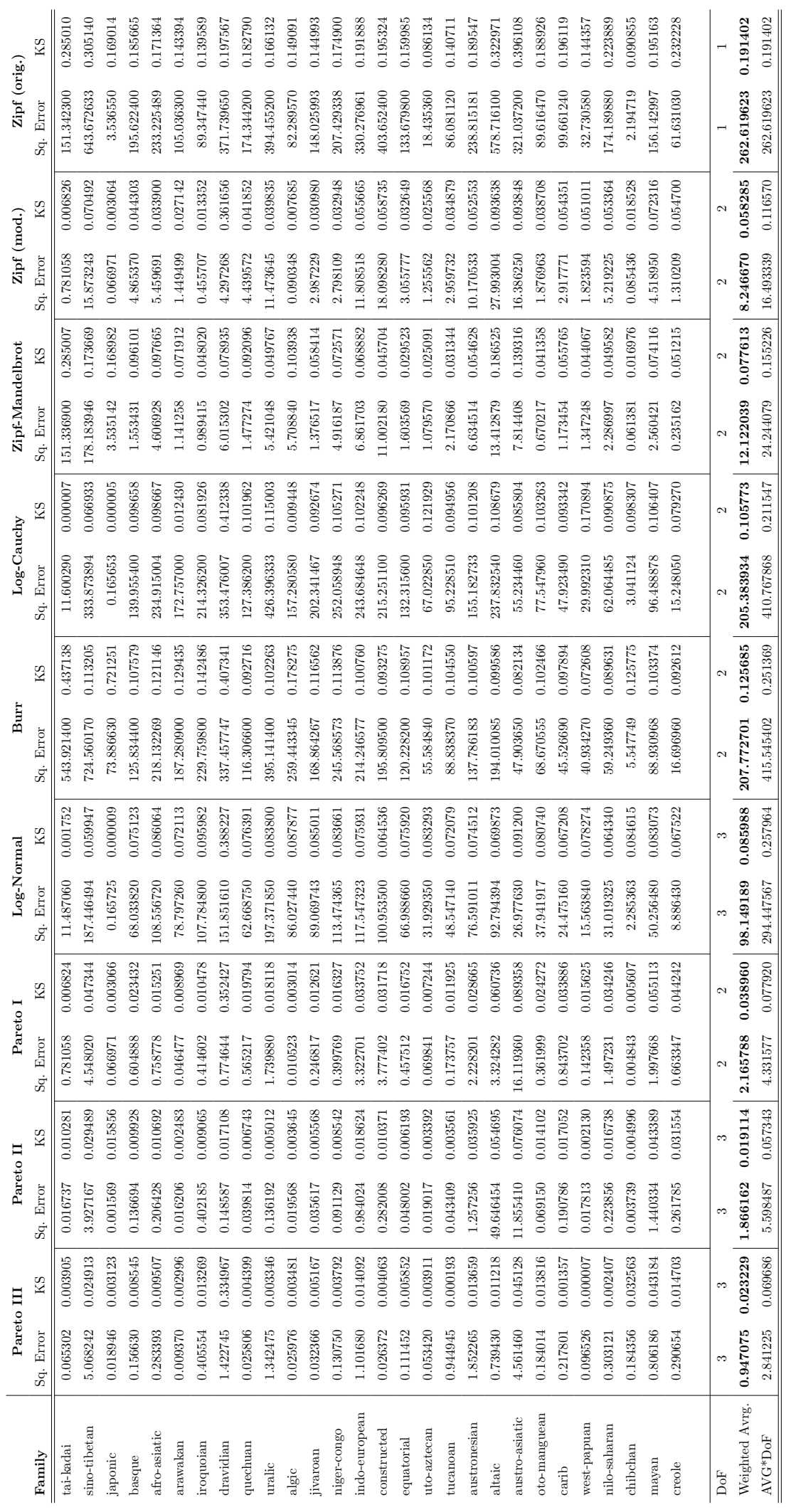

Table 3: Distribution performances grouped by language family. 


\section{Conclusions}

We have tested a number of distributions to accurately capture word frequencies. In comparison to the more established Zipf's law the Pareto type distributions have performed far better, supported by the Log-Log plots as well as the number of error measures we have calculated.
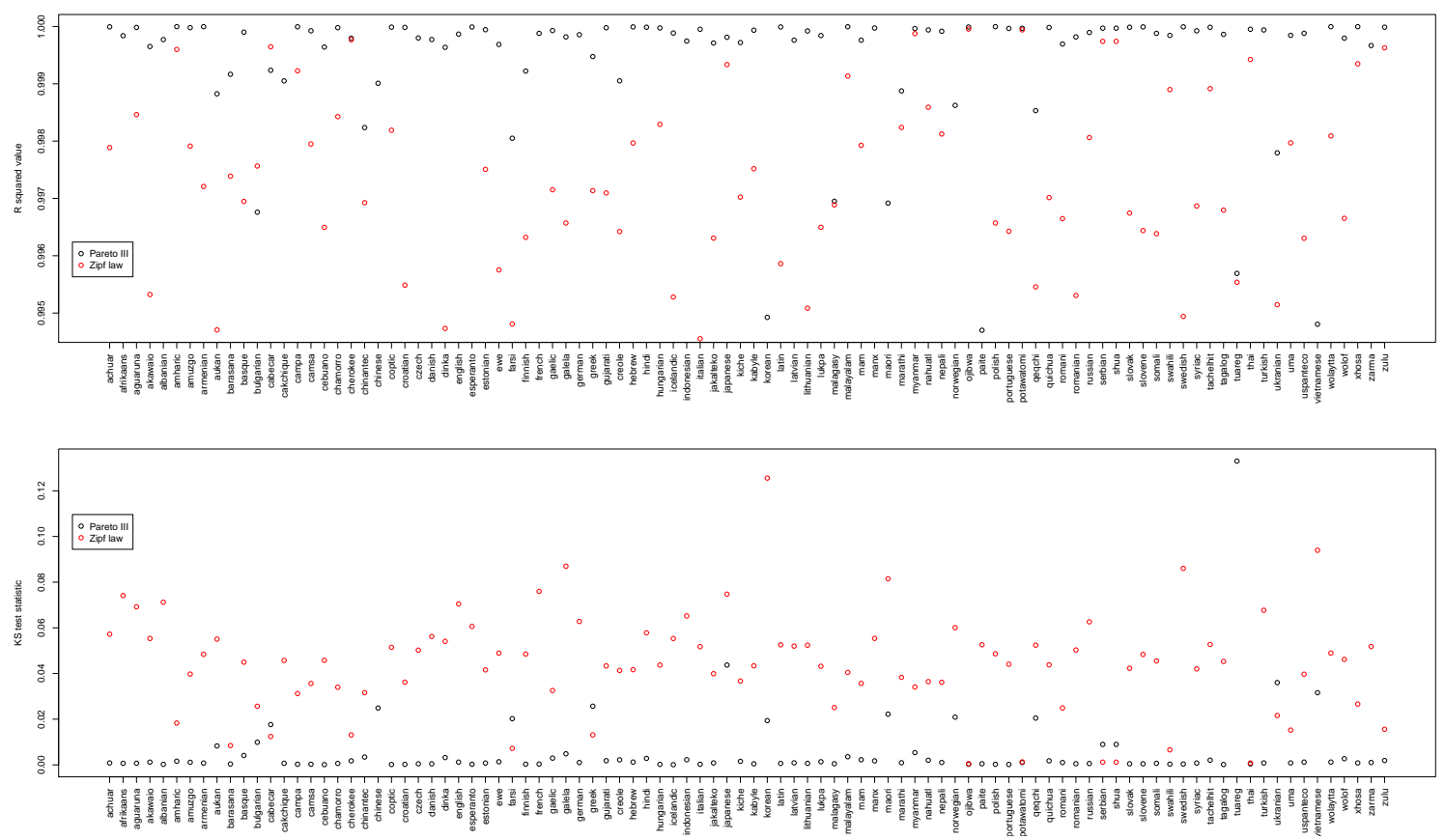

Figure 5: The K-S statistic and R squared measure for all languages compared.

The greater flexibility of the newly introdced distribution expectedly resulted in a better fit. Even though the Pareto distributions possess more parameters, the improvement of the fit is disproportionate to the increased complexity. The various incarnations of the Zipf law are in essence a rigid line on a Log-Log plot, which works somewhat for the body of the samples, but the Pareto-type distributions greatly improve the tail fit on boths ends of the frequencies.

It is evident to us that the models in this note are superior to Zipf's law and could greatly improve future modeling approaches.

\section{Acknowledgments}

We would like to express our thanks to Ali Mehri (University of Babol) for kindly providing the data sets used in [18] and for useful comments. The authors would also like to thank the Editor and the three referees for careful reading and comments which greatly improved the paper. 


\section{References}

[1] D. R. Amancio, Authorship recognition via fluctuation analysis of network topology and word intermittency. Journal of Statistical Mechanics, 2015, doi:10.1088/1742$5468 / 2015 / 03 / \mathrm{P} 03005$

[2] D. R. Amancio, E. G. Altmann, D. Rybski, O. N. Oliveira Jr, L. F. Costa, Probing the statistical properties of unknown texts: Application to the Voynich manuscript. PLoS ONE, 8, e67310, 2013, doi: 10.1371/journal.pone.0067310

[3] D. R. Amancio, O. N. Oliveira Jr, L. F. Costa, Structure-semantics interplay in complex networks and its effects on the predictability of similarity in texts. Physica A, 391, 4406-4419, 2012.

[4] F. Auerbach, Das gesetz der bevolkerungskonzentration. Petermanns Geographische Mitteilungen, 59, 74-76, 1913.

[5] R. H. Baayen, Word Frequency Distributions. Springer Science and Business Media, 2002.

[6] https://www.browserling.com/tools/word-frequency

[7] R. F. Cancho, B. Elvevag, Random texts do not exhibit the real Zipf's law-like rank distribution. PLoS ONE, 5, e9411, 2010, doi: 10.1371/journal.pone.0009411

[8] R. F. Cancho, R. V. Sole, Two regimes in the frequency of words and the origins of complex lexicons: Zipf's law revisited. Journal of Quantitative Linguistics, 8, 165, 2001.

[9] R. F. Cancho, R. V. Sole, Least effort and the origins of scaling in human language. Proceedings of the National Academy of Sciences of the United States of America, 100, 788-791, 2003.

[10] A. Clauset, C. R. Shalizi, M. E. J. Newman, Power-law distributions in empirical data. SIAM Review, 51, 661, 2009.

[11] C. Collodi, Pinocchio First Edition, 1883.

[12] M. Gerlach, E. G. Altmann, Stochastic model for the vocabulary growth in natural languages. Physical Review X, 3, 021006, 2013.

[13] http://homepages.inf.ed.ac.uk/s0787820/bible/

[14] B. B. Mandelbrot, An informational theory of the statistical structure of languages. In: Communication Theory, pp. 486-502, 1955.

[15] C. D. Manning, H. Schutze, Foundations of Statistical Language Processing. MIT Press, 1999.

[16] K. Marx Das Kapital. Kritik der politischen Ökonomie Volume I, 1867.

[17] A. P. Masucci, G. J. Rodgers, Differences between normal and shuffled texts: Structural properties of weighted networks. Advances in Complex Systems, 12, 2009, doi: 10.1142/S0219525909002039

[18] A. Mehri, M. Jamaati, Variation of Zipf's exponent in one hundred live languages: A study of the Holy Bible translations. Physics Letters A, 381, 2470-2477, 2017. 
[19] R Development Core Team, R: A Language and Environment for Statistical Computing. R Foundation for Statistical Computing, Vienna, Austria, 2017.

[20] A. de Saomt-Exupery, Le Petit Prince. 1947.

[21] J. R. Williams, J. P. Bagrow, C. M. Danforth, P. S. Dodds, Text mixing shapes the anatomy of rank-frequency distributions. Physical Review E, 91, 052811, 2015.

[22] http://www.writewords.org.uk/wordcount.asp/

[23] G. K. Zipf, Human Behavior and the Principle of Least Effort. Addison-Wesley, Massachusetts, 1949. 


\section{Appendix}
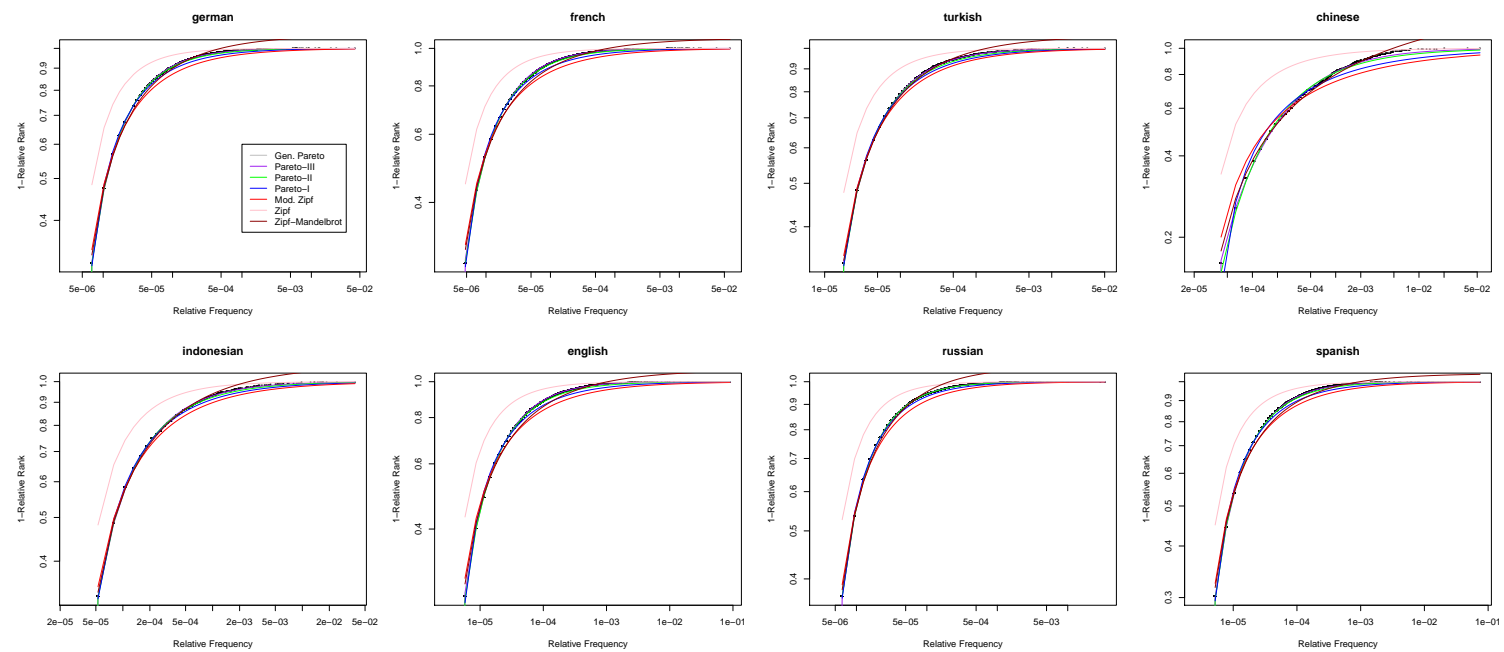

Figure 6: Log-Log CDF plot of word relative frequency versus relative rank for "Das Kapital".
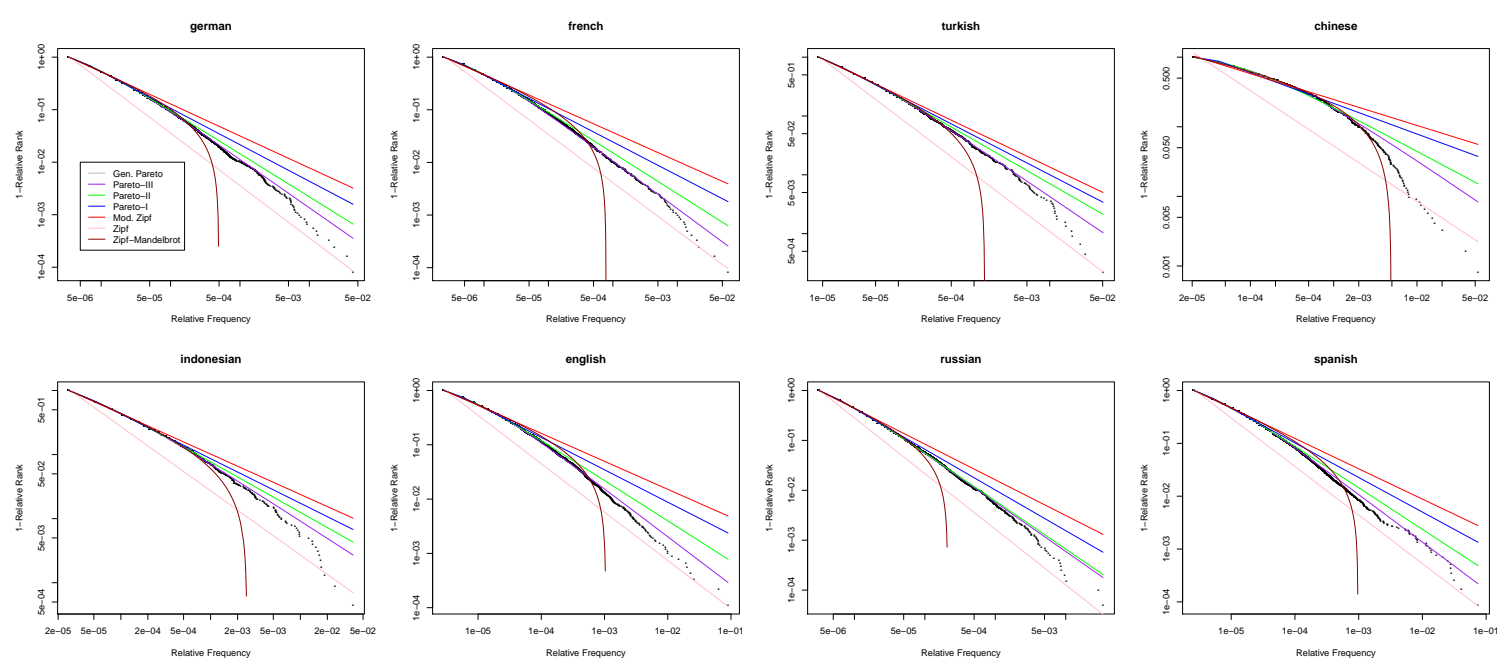

Figure 7: Log-Log CDF plot of word relative frequency versus inverse relative rank for "Das Kapital". 

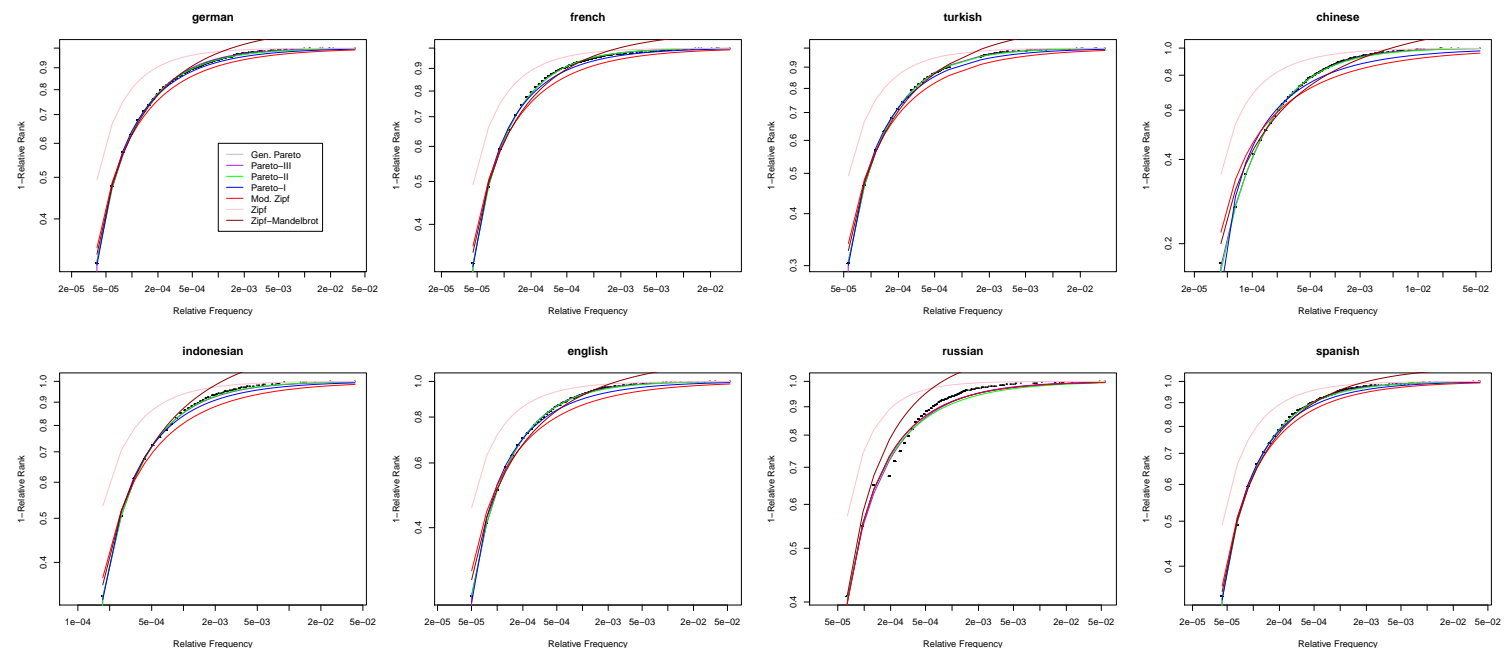

Figure 8: Log-Log CDF plot of word relative frequency versus relative rank for "Pinocchio".
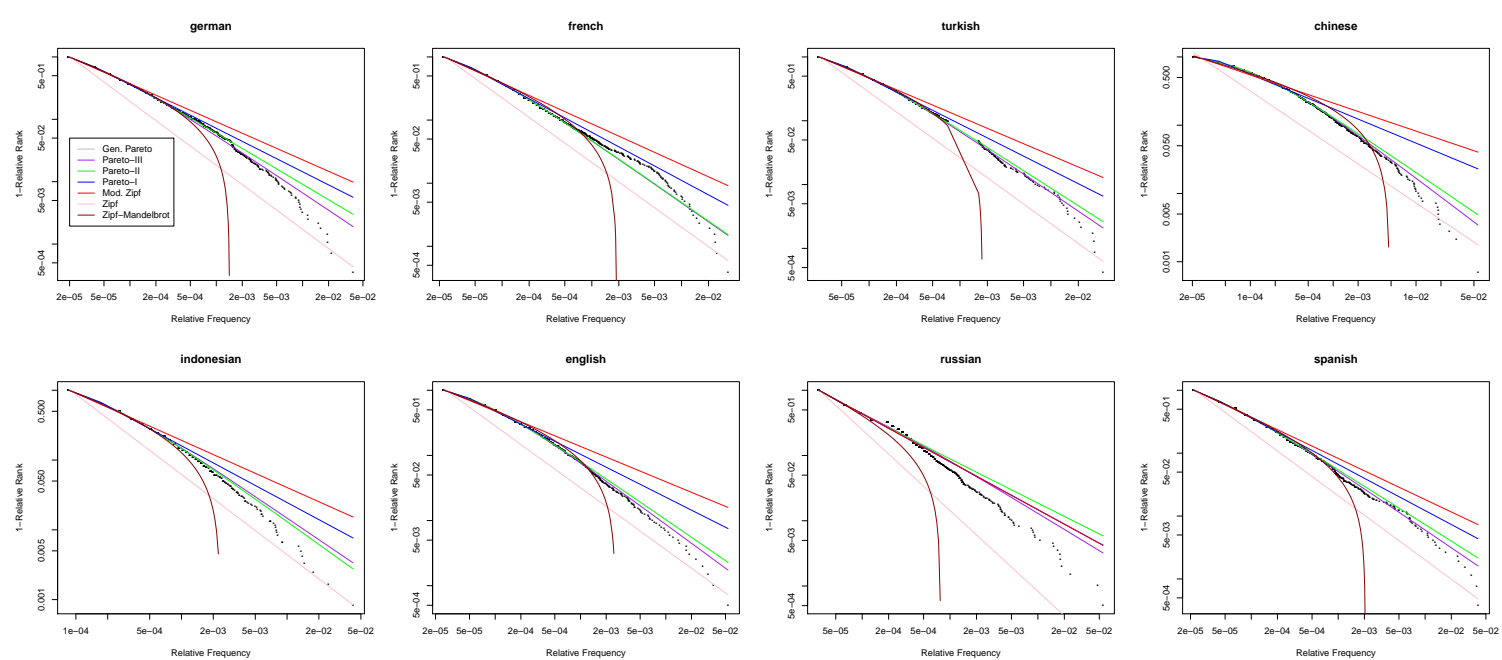

Figure 9: Log-Log CDF plot of word relative frequency versus inverse relative rank for "Pinocchio". 

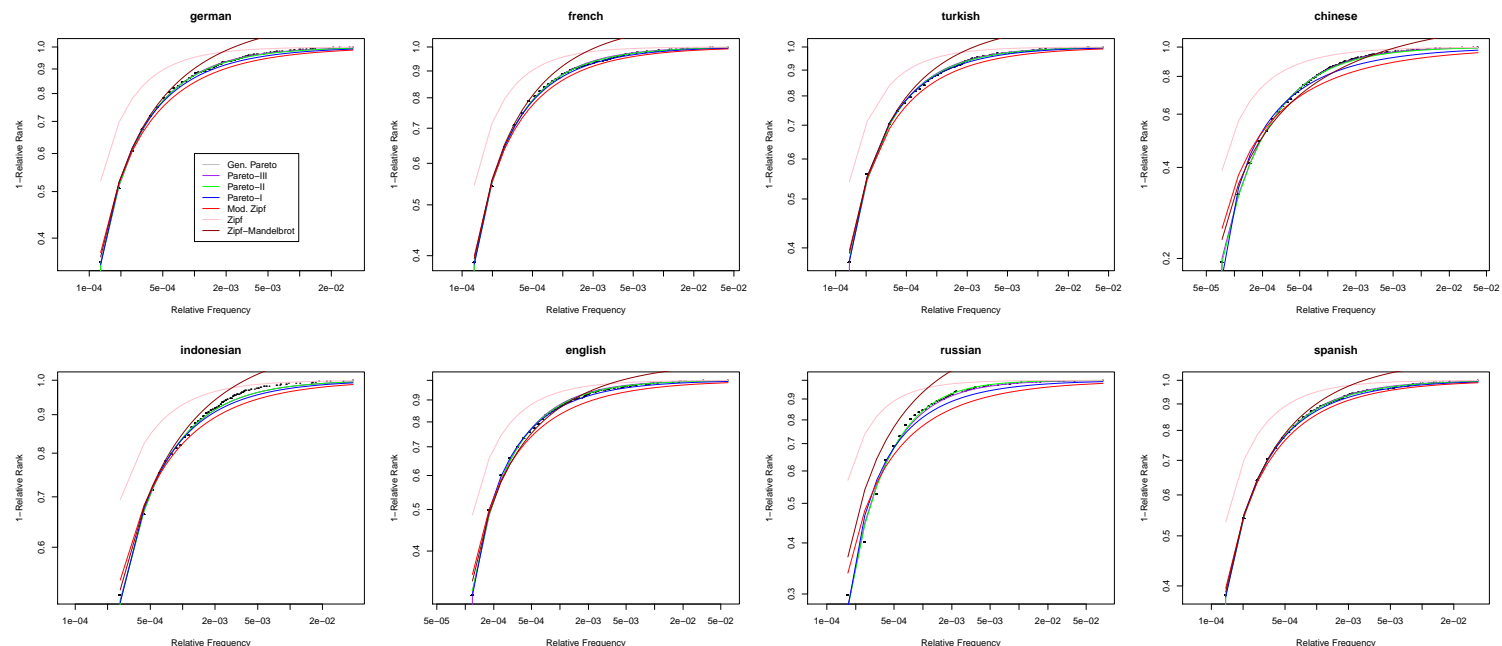

Figure 10: Log-Log CDF plot of word relative frequency versus relative rank for "The little Prince".
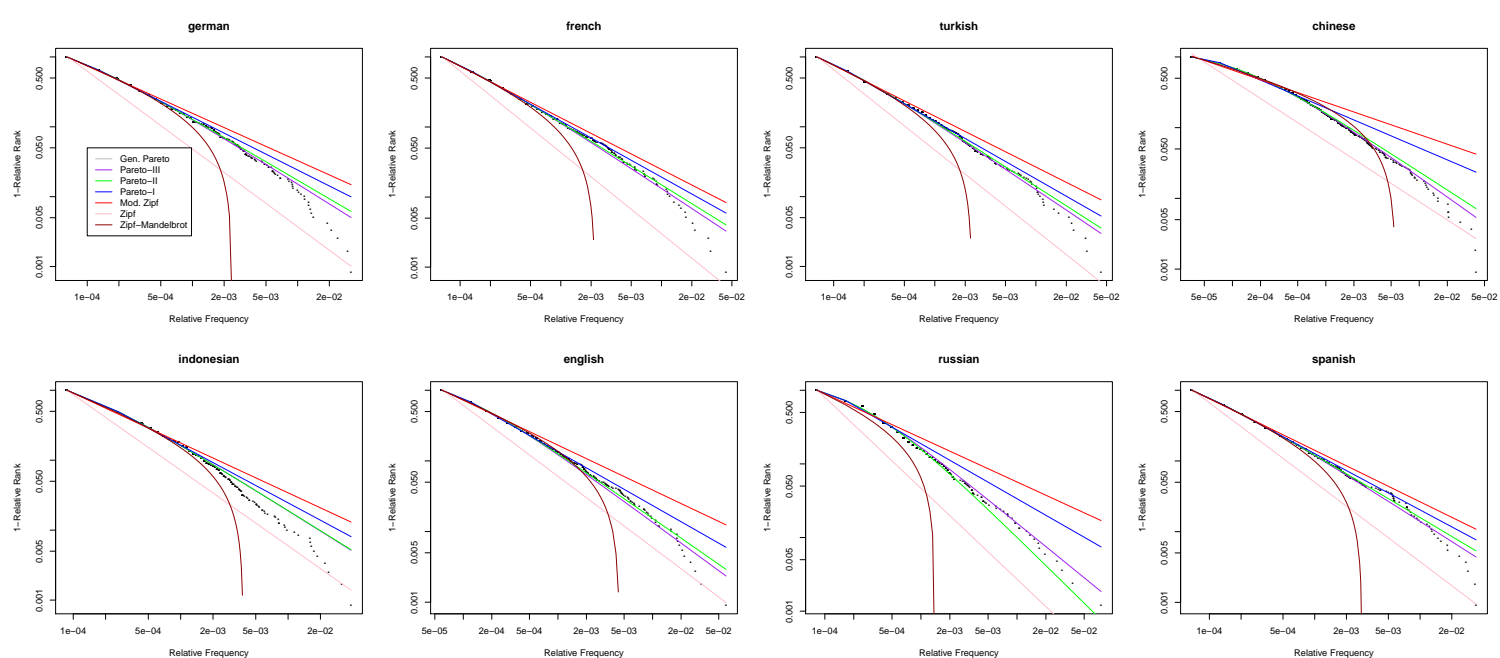

Figure 11: Log-Log CDF plot of word relative frequency versus inverse relative rank for "The little Prince". 


\section{Language Families}

\begin{tabular}{|c|c|c|c|}
\hline afro-asiatic & chibchan & danish & lukpa \\
\hline hebrew & cabecar & spanish & \\
\hline amharic & & italian & nilo-saharan \\
\hline coptic & constructed & french & dinka \\
\hline tachelhit & esperanto & nepali & zarma \\
\hline syriac & & afrikaans & \\
\hline wolaytta & creole & german & oto-manguean \\
\hline tuareg & aukan & portuguese & amuzgo \\
\hline somali & creole & swedish & chinantec \\
\hline kabyle & & norwegian & \\
\hline arabic & $\begin{array}{l}\text { dravidian } \\
\text { telugu }\end{array}$ & $\begin{array}{l}\text { manx } \\
\text { english }\end{array}$ & $\begin{array}{l}\text { quechuan } \\
\text { quichua }\end{array}$ \\
\hline algic & malayalam & gujarati & \\
\hline $\begin{array}{l}\text { potawatomi } \\
\text { ojibwa }\end{array}$ & kannada & romani & $\begin{array}{l}\text { sino-tibetan } \\
\text { chinese }\end{array}$ \\
\hline $\begin{array}{l}\text { altaic } \\
\text { turkish }\end{array}$ & $\begin{array}{l}\text { equatorial } \\
\text { camsa }\end{array}$ & $\begin{array}{l}\text { iroquoian } \\
\text { cherokee }\end{array}$ & $\begin{array}{l}\text { myanmar } \\
\text { paite }\end{array}$ \\
\hline korean & $\begin{array}{l}\text { indo-european } \\
\text { farsi }\end{array}$ & $\begin{array}{l}\text { japonic } \\
\text { japanese }\end{array}$ & $\begin{array}{l}\text { tai-kadai } \\
\text { thai }\end{array}$ \\
\hline arawakan & latvian & & \\
\hline campa & $\begin{array}{l}\text { ukranian } \\
\text { armenian }\end{array}$ & $\begin{array}{l}\text { jivaroan } \\
\text { shuar }\end{array}$ & $\begin{array}{l}\text { tucanoan } \\
\text { barasana }\end{array}$ \\
\hline $\begin{array}{l}\text { austro-asiatic } \\
\text { vietnamese }\end{array}$ & $\begin{array}{l}\text { lithuanian } \\
\text { croatian } \\
\text { latin }\end{array}$ & $\begin{array}{l}\text { aguaruna } \\
\text { achuar }\end{array}$ & $\begin{array}{l}\text { uralic } \\
\text { hungarian }\end{array}$ \\
\hline austronesian & hindi & mayan & finnish \\
\hline chamorro & albanian & kiche & estonian \\
\hline indonesian & polish & qeqchi & \\
\hline uma & czech & uspanteco & uto-aztecan \\
\hline malagasy & slovene & cakchiquel & nahuatl \\
\hline cebuano & russian & jakalteko & \\
\hline $\begin{array}{l}\text { tagalog } \\
\text { maori }\end{array}$ & $\begin{array}{l}\text { bulgarian } \\
\text { greek }\end{array}$ & mam & $\begin{array}{l}\text { west-papuan } \\
\text { galela }\end{array}$ \\
\hline & slovak & niger-congo & \\
\hline basque & romanian & zulu & \\
\hline basque & icelandic & xhosa & \\
\hline & serbian & ewe & \\
\hline carib & marathi & swahili & \\
\hline akawaio & gaelic & wolof & \\
\hline
\end{tabular}

Table 4: Grouping of all analysed languages into their respective families 


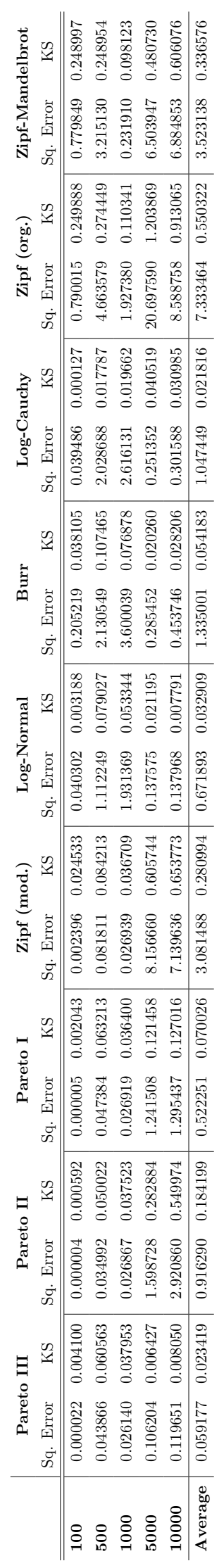

Table 5: Error Measures for randomly generated texts of different lengths. 